\title{
Influence of Calcium Phosphates on the Degradation of Poly Lactic Acid for Medical Implants
}

\section{Journal Article}

Author(s):

Ruffieux, K.; Dell'Agosti, A.; Riesen, B.; Wintermantel, E.

Publication date:

1996

Permanent link:

https://doi.org/10.3929/ethz-b-000422544

Rights / license:

In Copyright - Non-Commercial Use Permitted

Originally published in:

Biomedizinische Technik 41(s1), https://doi.org/10.1515/bmte.1996.41.s1.420 


\title{
Influence of Calcium Phosphates on the Degradation of Poly Lactic Acid for Medical Implants
}

\author{
K. Ruffieux, A. Dell'Agosti, B. Riesen, E. Wintermantel \\ Chair of Biocompatible Materials Science and Engineering, Dept. of Materials Science \\ Swiss Federal Institute of Technology, Zürich \\ Wagistr. 23, 8952 Schlieren, Switzerland
}

\section{INTRODUCTION}

In different in vivo-studies, poly lactic acid (PLA) was used for numerous orthopedic applications. In many cases, its use lead to complications, often requiring surgical removal of the implants. Two possible explanations are being discussed in the literature [1-4]: crystallites remaining after degradation of the amorphous phase and the acidic degradation of PLA. Therefore, in this study, 2 different calcium phosphates (Ca-P) were added to intrinsically amorphous poly-D,L-lactic acid (PDLLA) to neutralize acidic degradation products.

\section{MATERIALS AND METHODS}

Rods $(2 \times 3 \times 20 \mathrm{~mm})$ of high molecular weight PDLLA (Resomer R207, Boehringer Ingelheim, Mw $\approx 200^{\prime} 000$ $\mathrm{g} / \mathrm{mol}$ ) with and without particles of $\beta$-tri-calcium phosphate (TCP) and calcium hydrogen phosphate (CHP) (Fluka, $\mathrm{x}_{50}=2.2 \mu \mathrm{m}$ ) were injection molded at $140^{\circ} \mathrm{C}$. The polymer was pre-dried at $40^{\circ} \mathrm{C}$ for 48 hours and at $70^{\circ} \mathrm{C}$ for 12 hours. Small amounts of Ca-P were continously added during the injection molding process.

The in vitro degradation was performed using Ringer's solution (pH 7.4) with an isotonic composition similar to body fluids at $37^{\circ} \mathrm{C}$. The samples were not shaken and the medium was not exchanged during the test. Degradation of 3 to 5 samples was monitored up to 40 weeks. Parameters tested were: mass change, $\mathrm{pH}$-value (Metrohm $\mathrm{pH}$-meter), mechanical properties by 3-point bending ( $1 \mathrm{~mm} / \mathrm{min}$., support length $13 \mathrm{~mm}$ ). Molecular weight was determined by size exclusion chromatography (Knauer, Germany, PLgel $5 \mathrm{~mm}$ mixed-C column, THF, $23^{\circ} \mathrm{C}$ ) using PS-standards (Polymer Laboratories) as reference. The surface morphology and the fracture behavior of the samples were examined using SEM.

\section{RESULTS AND DISCUSSION}

During the in vitro test, PDLLA samples absorbed $8 \%$ (weight), PDLLA/TCP 18\% and PDLLA/CPH $28 \%$ water within 32 weeks. Afterwards, weight decreased with time. The water uptake correlated to the observed swelling of the samples.

Molecular weight measurements showed that rods containing TCP- or CHP-particles degraded slower than the pure PDLLA-rods (figure 1) due to a neutralization of acidic end groups which could otherwise autocatalyze the degradation of PLA [5]. PDLLA/TCP degraded faster than
PDLLA/CHP, probably due to the lower solubility of TCP (ca. $5.10^{-4} \mathrm{Ca}^{+} / \mathrm{mol}^{-1} \mathrm{~L}^{-1}$ ) as compared to CHP (ca. $\left.10^{-3} \mathrm{Ca}^{+} / \mathrm{mol} \cdot \mathrm{L}^{-1}\right)[6]$.

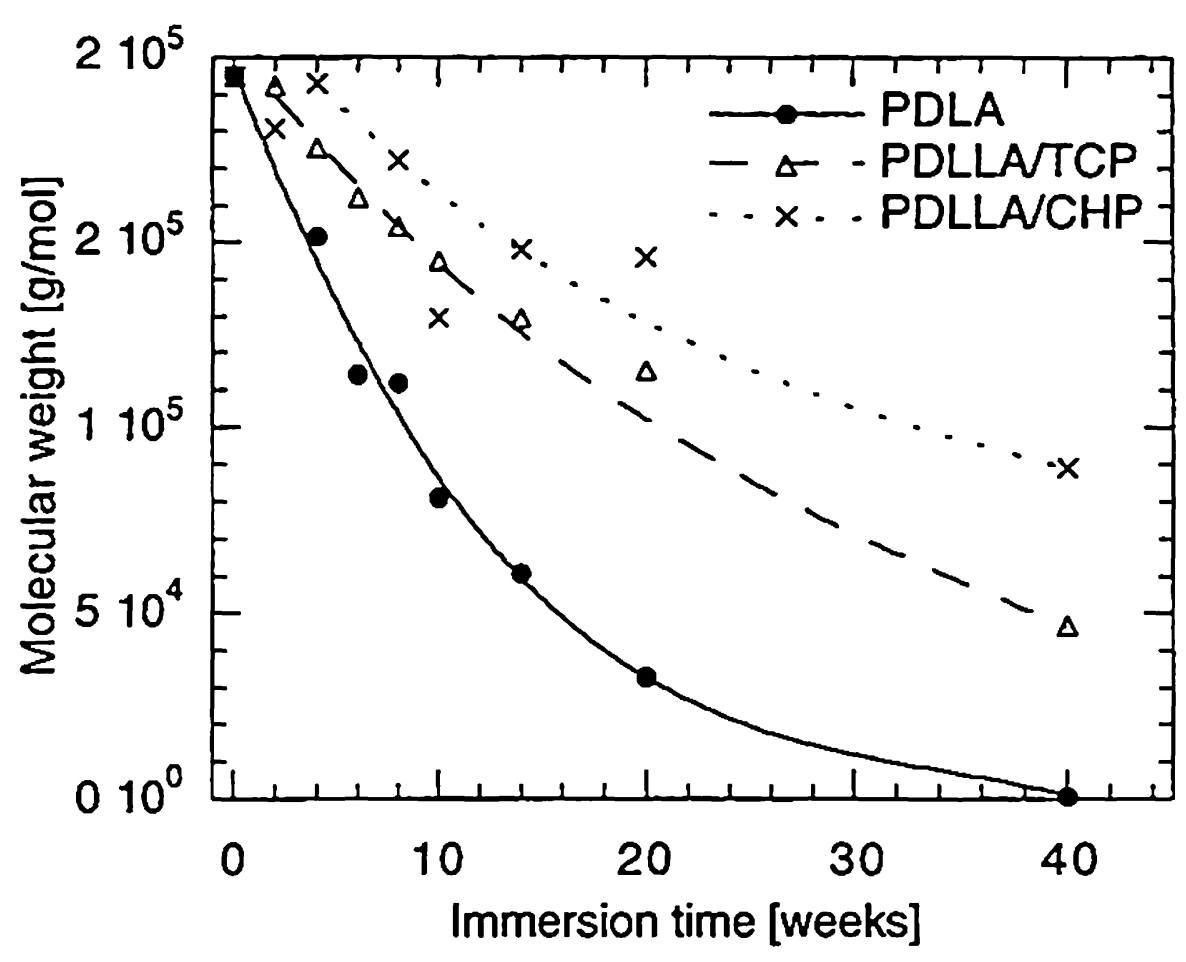

Figure 1: Decrease of the molecular weight as a function of the immersion time. Samples of pure $P D L L A$ degraded at a faster rate than samples containing Ca-P-particles.

The initial strength of ca. $95 \mathrm{MPa}$ for the samples with Ca-P-particles was reduced immediately after immersion, probably due to water uptake and to a possible particle / polymer debonding (figure 2). While the pure PDLLAsamples showed a strength retention during the first 10 weeks followed by a fast decrease in strength, the samples with Ca-P showed a slow linear decrease during the observation period.

The uptake of water also reduced the stiffness of the samples with TCP-particles within the first 2 weeks from 3700 to $2800 \mathrm{MPa}$, after which a linear decrease to ca. $500 \mathrm{MPa}$ within 40 weeks occured. A stiffening effect from the initial water uptake could be measured for the pure PDLLA samples and the ones with CHP. Afterwards, a linear decrease could be observed. PDLLA samples had a Young's modulus of $500 \mathrm{MPa}$ after 20 weeks, whereas PDLLA/CHP showed similar behaviour to the PDLLA/TCP-samples. Standard deviations of up to $250 \mathrm{MPa}$ were measured.

$\mathrm{pH}$ of the medium surrounding the samples was initially 8.1 , which was reduced to 7.4 within the first 4 weeks of observation (figure 3 ). The higher $\mathrm{pH}$ of the Ringer's solution was probably due to a loss of $\mathrm{CO}_{2}$ during storage. Up to 30 weeks, no significant change of $\mathrm{pH}$ 
could be observed. After 32 weeks $\mathrm{pH}$ of one of the PDLLA-samples fell to 2, whereas the other remained at 7.4. The decrease in $\mathrm{pH}$ could be correlated to an observed burst of the existing surface skin (compare with figure 4). After 40 weeks $\mathrm{pH}$ of all PDLLA samples dropped to 1.8 , opposed to the samples with $\mathrm{Ca}-\mathrm{P}$ where no lowering of the $\mathrm{pH}$ could be observed.

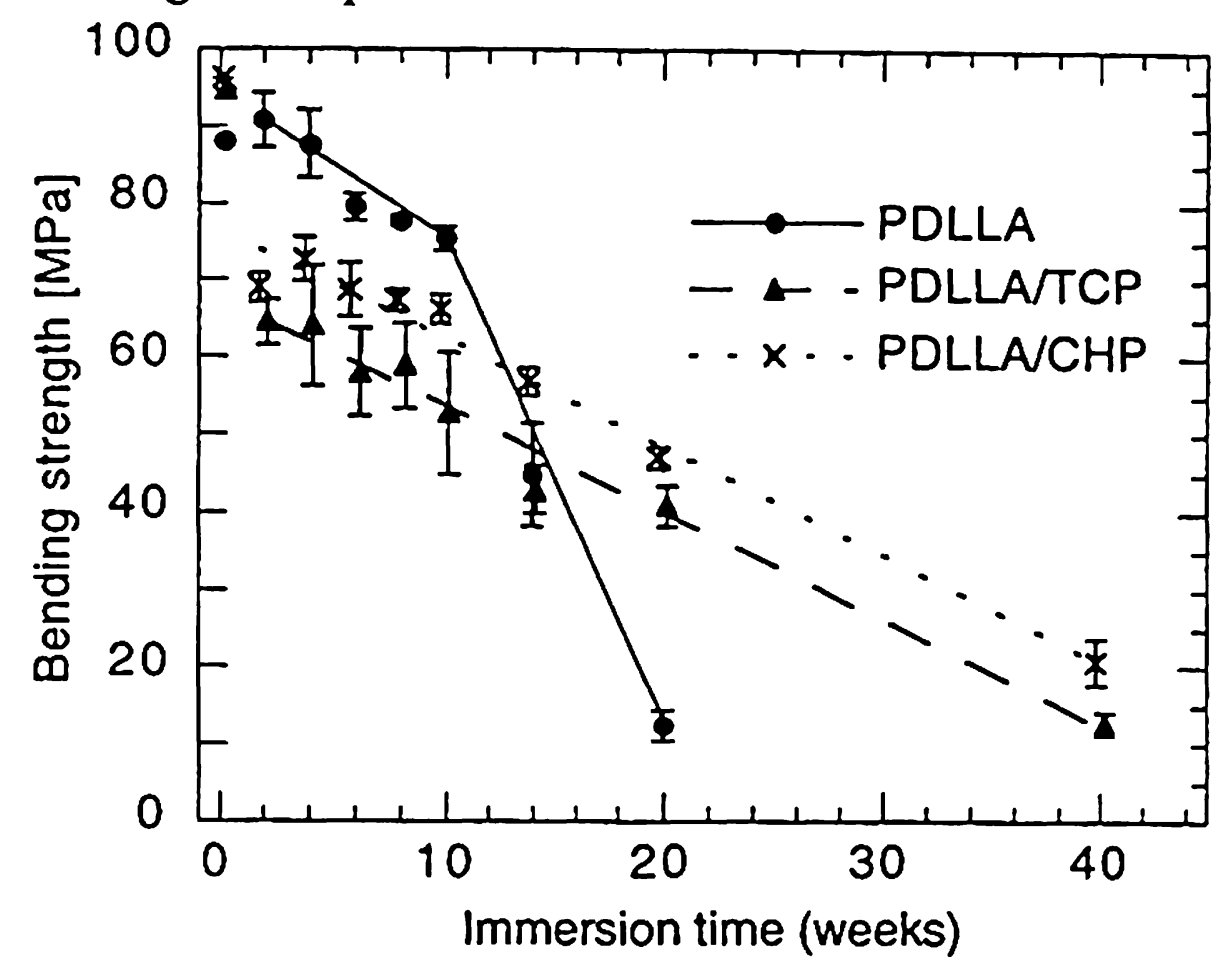

Figure 2: Bending strength of the test bars measured in a 3-point bending test. After 10 weeks, pure PDLLA samples showed a rapid decrease in strength, whereas samples with $C a-P$ showed a slower, linear decrease.

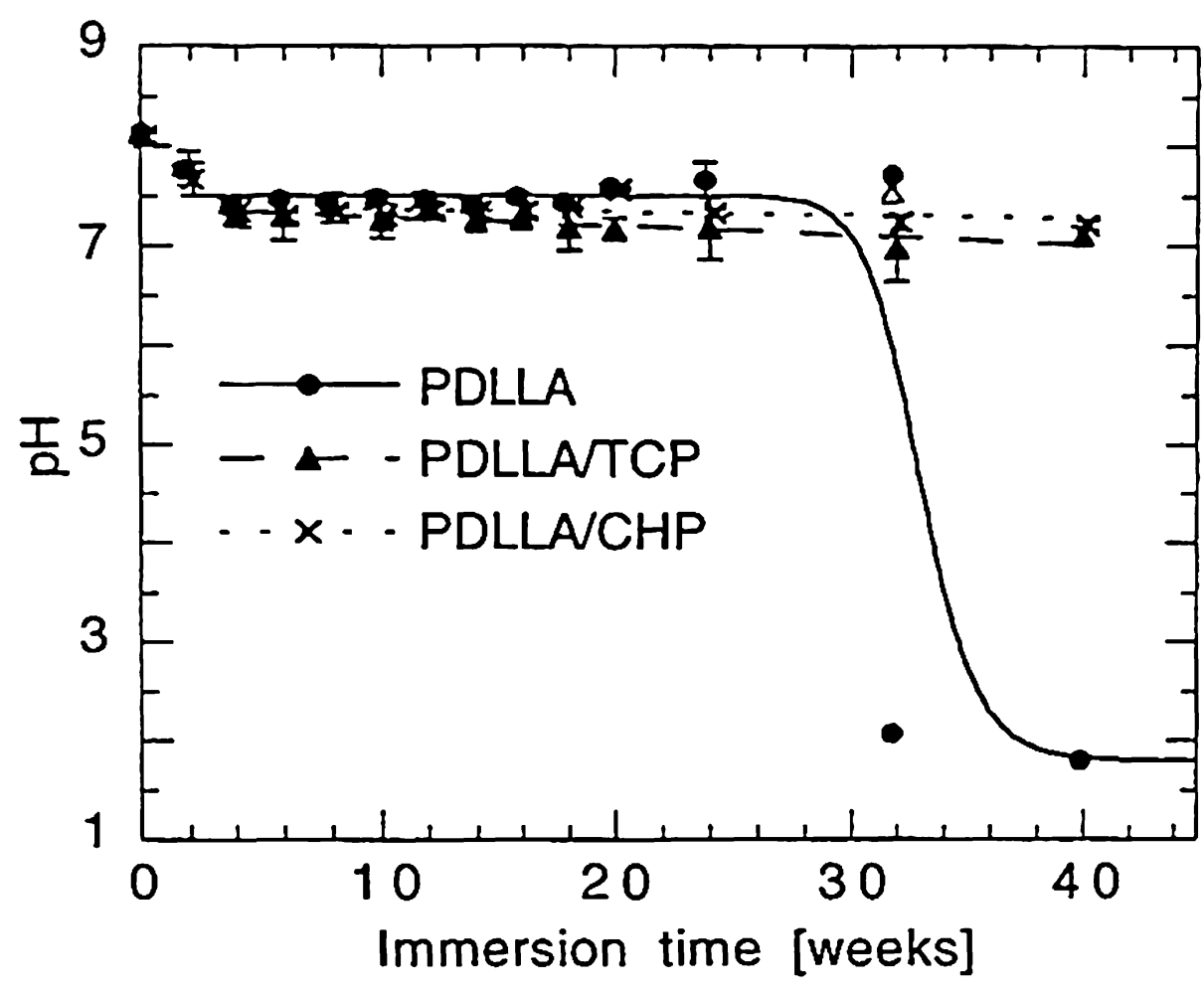

Figure 3: $p H$-values of the Ringer's solution during degradation of $P D L L A$ rods. $P H$ of rods containing TCP-or CHP-particles remained constant during 40 weeks, whereas $p H$ of the pure PDLLA-rods was lowered to 2 after 32 weeks.

SEM analysis of the samples revealed the formation of a skin $(6 \mu \mathrm{m})$ by the injection molding process (figure 4$)$. Furthermore, degradation products may be released more easily from the surface layer into the surrounding medium than from the center of the samples. Therefore, degradation in the outer zones of PLA-samples is slower than in the center. It is assumed that this skin may have acted as a diffusion barrier for degradation products. In
PDLLAVTCP samples, small cracks in the surface of the specimen could be observed after 10 weeks of degradation (figure 5). These cracks were probably initiated by the observed swelling of the samples. Pure PDLLA samples did not show any such small cracks during the observation time. For the pure PDLLA samples, a bursting of the outer skin was observed.

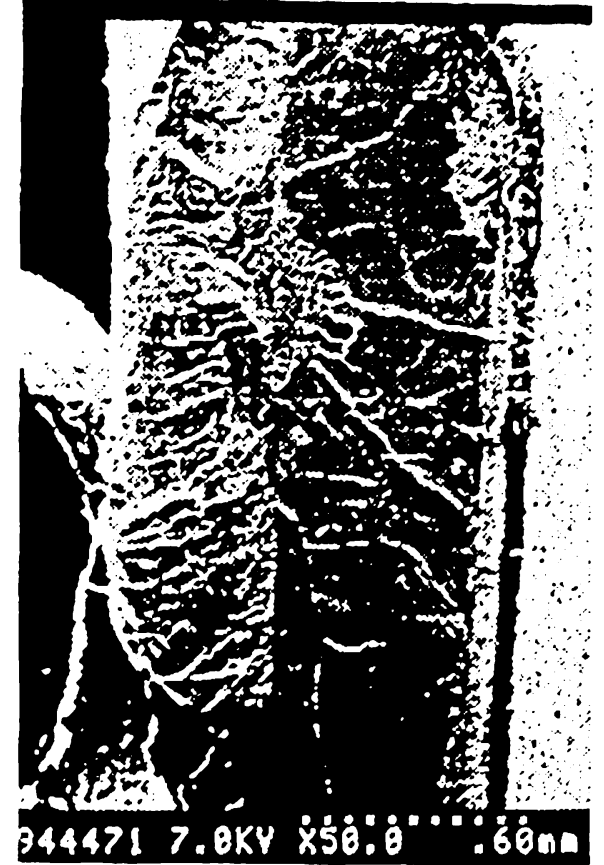

Figure 4:

Cross section (50x) of a PDLLA sample before degradation. A skin with a thickness of about $6 \mu \mathrm{m}$ can be seen, which may be a result of the injection molding process.

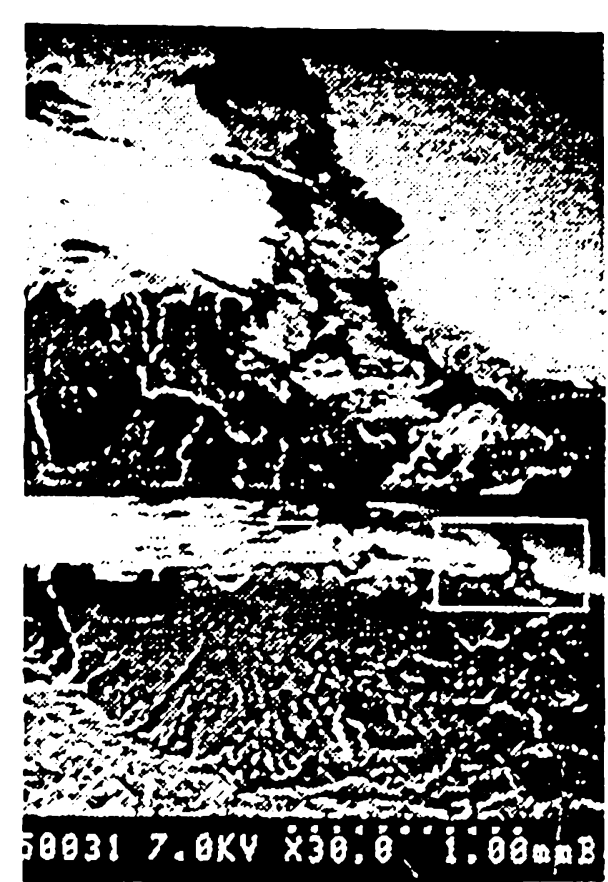

Figure 5:

Surface area $(30 \times / 300 x)$ of a PDLLA/TCP sample after 10 weeks of degradation in vitro. The skin shows cracks, which may increase the release of degradation products into the surrounding medium.

\section{CONCLUSIONS}

The addition of Ca-P particles to PDLLA resulted in a slower degradation behavior as compared to pure PDLLA. It is assumed that this is due to the neutralization of acidic groups which result from the polymer degradation. This hinders the autocatalytic stimulation of the degradation by these groups. A burst release of acidic degradation products could not be seen for samples with $\mathrm{Ca}-\mathrm{P}$ during observation time. From these conclusions, it is suggested that the addition of tri-calcium phosphate or calcium hydrogen phosphate results in a more physiological degradation behavior of implants made of amorphous poly lactic acid.

\section{REFERENCES}

1. H. Pistner et al.; Biomat., 1993, Vol. 14(9), 671-677.

2. E. Bergsma et al.; J. of oral and maxillofac. surg., 1993, Vol. 51(6), 666-670.

3. O. Böstmann et al.; J. Bone Joint Surgery, 1990, Vol. 72-B, 592-596.

4. K. Ruffieux et al.; 7th Int. Conf. on Pol. in Med. and Surg., 1-3.9.1993, Noordwijkerhout, NL, 327-335.

5. M. Vert et al.; J. of Mat. Sci.: Mat. in Med., 1992, Vol. 3, 432-446.

6. G. Bauer et al.; cfi/DKG Berichte, 1989, Vol. 66(1/2), 23-27. 